\title{
1 Luteolin transforms the BMDM polarity to regulate the expression of
}

2 inflammatory factors

3 Shuxia Wang, ${ }^{\mathrm{a}}$ Shuhang Xu, ${ }^{\mathrm{b}}$ Meng Cao, ${ }^{\mathrm{c}}$; Jing Zhou, ${ }^{\mathrm{c}}$ Xiaodong Mao, ${ }^{\mathrm{b}}$ Xiaoming Yao, ${ }^{\mathrm{a}}$ Chao

4 Liu, ${ }^{\mathrm{b}}$

$5{ }^{\mathrm{a}}$ Clinical Laboratory, Affiliated Hospital of Integrated Traditional Chinese and Western Medicine,

6 Nanjing University of Chinese Medicine; Jiangsu Province Academy of Traditional Chinese

7 Medicine, Nanjing, Jiangsu, China

${ }^{\mathrm{b}}$ Research Center of Endocrine and Metabolic Diseases, Affiliated Hospital of Integrated

9 Traditional Chinese and Western Medicine, Nanjing University of Chinese Medicine; Jiangsu

10 Province Academy of Traditional Chinese Medicine, Nanjing, Jiangsu, China

11 c Affiliated Hospital of Integrated Traditional Chinese and Western Medicine, Nanjing University

12 of Chinese Medicine, Nanjing, Jiangsu, China

13 Address correspondence to Xiaoming Yao and Chao Liu: Yaoxm73@163.com;

14 profliuchao@163.com

15 ABSTRACT Macrophage are indispensable regulator cells in inflammatory response.

16 Macrophage polarization and its secreted inflammatory factors have affinity with the outcomes of

17 inflammation. Luteolin, a flavonoid abundant in plants has anti-inflammatory activity, but whether luteolin can manipulate M1/M2 polarization of BMDM to suppress inflammation is still veiled.

19 The purpose of this study was to observe the effects of luterolin on the polarity of BMDM derived 
luteolin regulating the BMDM polarity. M1-polarized BMDM were induced by LPS+IFN- $\gamma$, M2-polarization were stimulated with IL-4. BMDM morphology was observed by laser confocal microscopy; levels of BMDM differentiation and CD11c or CD206 on membrane surface were assessed by FCM; mRNA and protein of M1/M2-type inflammatory factors were performed by qPCR and ELISA, respectively; the expression of p-STAT1 and p-STAT6 protein pathways was detected by Western-blotting. The isolated mouse bone marrow cells were successfully differentiated into BMDM, LPS+IFN- $\gamma$ induced BMDM M1-phenotype polarization, and IL-4 induced its M2-phenotype polarization. After M1-polarized BMDM treated with luteolin, M1-type pro-inflammatory factors including IL-6, TNF- $\alpha, \square$ iNOS, CD86 were down-regulated while M2-type anti-inflammatory factors including IL-10, Arg1, CD206 were up-regulated; the expression of M1-type surface marker CD11c decreased, nevertheless, M2-type marker CD206 increased; levels of inflammatory signaling protein p-STAT1 and p-STAT6 were attenuated and enhanced respectively. Our study suggests luteolin may transform BMDM polarity through p-STAT1/6 to regulate the expression of inflammatory mediators, thereby inhibiting inflammation. Naturally occurring luteolin hold promise as an anti-inflammatory and immunomodulatory agent.

KEYWORDS: cytokines, inflammation, BMDM polarization, luteolin

$$
\text { Inflammation is the immune system's response to invading pathogens, but aberrant }
$$
inflammation responses leads to a "cytokine storm" that makes patients sicker (1). Mounting evidences found that continuous and/or repeated inflammatory stimuli could also induce tumors (2). Therefore, the inflammatory response is a double-edged sword. If immune cells and pro-inflammatory cytokines are overproduced, cytokine cascades occur, called "cytokine storm" or termed as "inflammatory storm", leading to sepsis, acute respiratory distress syndrome (ARDS) 
and even multiple organ failure (MOF) (3). As well all know, pathogenic agents such as viral or bacterial infections incur the pathological process of sepsis which is characterized by an overwhelming generation of pro-inflammatory cytokines. Recently, global pandemic of coronavirus disease 2019 (COVID-19) suffering from severe acute respiratory syndrome coronavirus 2 (SARS-CoV-2) is also associated with macrophage hyperpolarization elicits "cytokine storms" and viral sepsis (4). Thus, the immunomodulatory therapy of inflammation is crucial for maintaining homeostasis (5-6).

Macrophages have been identified as critical effector cells in inflammatory/immune response and can be activated by pathogenic agents or inflammatory mediators to secrete various inflammatory factors. Meanwhile, heterogeneity and plasticity are hallmarks of macrophages, that is, M1-polarized (pro-inflammatory) macrophages and M2-polarized (anti-inflammatory) macrophages. Pathogens infection can polarize macrophages to M1-phenotype, produce high level of pro-inflammatory cytokines such as IL- 6 and TNF- $\alpha$, or effector molecules iNOS and surface markers CD11c or CD86, exert a pro-inflammatory effect and defense against pathogens. Conversely, IL-4 or TGF- $\beta$ induced M2-phenotype macrophages mainly express anti-inflammatory cytokine IL-10, effector molecule Arginase (Arg) 1 and surface marker CD206, contribute to hinder inflammation (7). Normally, the M1/M2 polarization of macrophages maintains a dynamic equilibrium. When virulent bacteria or viral infections or overmuch inflammatory molecules irritation, this balance is disrupted, excessive M1 polarization of macrophages will generate redundant inflammatory factors, causing systemic inflammatory response syndrome (SIRS, namely sepsis) and MOF (8). Therefore, it is particularly vital to skew 
response and promote tissue remodeling.

At present, most anti-inflammatory agents are glucocorticoids, antibiotics or antivirals. Hormones not only produce immunosuppression, but also induce secondary infections and prolong the disease course or other side effects. Antibiotics or antiviral drugs only kill the pathogen, and that antibiotics lyse the bacteria while killing the bacteria, releasing more toxins to induce "cytokine storms", further exacerbating the inflammatory response and promoting the promoting factor to the development of sepsis. In this regard, natural anti-inflammatory immune drugs extracted from plants have multi-effect regulation and less toxic, and especially have obvious benefits in suppressing inflammation. Therefore, it is urgent to seek natural compounds with high efficiency and low toxicity as anti-inflammatory immune agents.

Luteolin (Lut) is a flavonoid, mainly exists in fruits, vegetables and Chinese herbs, which has anti-hyperlipidemia (9), antitumor (10), anti-inflammation and immunoregulation (11). Studies showed that luteolin also has antiviral effects against Influenza A virus or dengue virus by interfering with coat protein (12-13). Recently research indicated that active ingredients of Chinese medicines including luteolin, quercetin etc. could manage COVID-19 by targeting on AEC2 and 3CL protein and dampen inflammatory mediators without side effects and have achieved significant clinical efficacies (14). Our previous investigation found that luteolin can regulate the expression of inflammatory factors in macrophages and play an anti-inflammatory role (7), but whether luteolin can regulate the polarization of macrophages and its molecular mechanism is still veiled. In this research, bone marrow cells isolated from C57BL/6 mice were induced to differentiate into bone marrow-derived macrophages (BMDM) for investigating the effects of luteolin on the M1/2 polarization of BMDM and the expression of inflammatory factors 
so as to explore the underlying mechanism. Our present findings preliminary provided luteolin could be a future perspective for the natural anti-inflammatory agent in prevention and treatment of sepsis.

\section{MATERIALS AND METHODS}

Mice. 6-week-old C57BL/6 mice (weighting 18-22 g) were provided from animal experimental center of affiliated hospital of integrated traditional Chinese and Western medicine, Nanjing University of Chinese medicine (Nanjing, China). Mice were maintained under specific pathogen-free conditions and in accordance with protocols approved by the National Institute of Health Guide for Care (Ethics Number: AEWC-20181019-53).

Isolation and culture of BMDM. C57BL/6 mice were sacrificed by cervical dislocation, and dissected under immersion in $75 \%$ ethanol (V/V). The epiphysis was cut after tibia and femur were separated, and the bone marrow cavity was rinsed with sterile PBS until the bone became white. The bone marrow washing solution was filtered with a $70 \mu \mathrm{m}$ mesh and transferred to a 50 $\mathrm{mL}$ centrifuge tube for cell collection. After lysis of red blood cells, bone marrow stem cells were resuspended in DMEM (Gibco, USA) containing $20 \mathrm{ng} / \mathrm{mL}$ M-CSF (R\&D System, USA) on 10-cm petri dishes and renewed the medium every other day. After 7 days incubation, cells were labeled with F4/80-PE fluorescently conjugated antibodies (1.0 $\mu \mathrm{L}$; eBioscience, USA) to identify the differentiation degree of mature BMDM by FCM (Millipore, USA).

Cell viability assay. BMDM were plated in 96 -well plates at a density of $2 \times 10^{4}$ cells/well in $200 \mu \mathrm{L}$ medium with confluence overnight. After that, the cells were exposed with LPS $(20 \mathrm{ng} / \mathrm{mL}$; 
and LPS plus IFN- $\gamma$-primed cells were administrated with luteolin (Sigma, USA) at 2.5 and 5.0 $\mu \mathrm{mol} / \mathrm{L}$ for $24 \mathrm{~h}$. Following treatment, cells were added with $20 \mu \mathrm{L}$ MTT solution $(5 \mathrm{mg} / \mathrm{mL}$; Sigma, MO. USA). $4 \mathrm{~h}$ later, culture medium was removed and crystals were dissolved with 150 $\mu \mathrm{L} /$ well DMSO. The absorption values were measured at $570 \mathrm{~nm}$.

The morphology of polarized BMDM. BMDM in logarithmic stage were cultivated in 6-well plates, and incubated overnight followed by treatment with LPS $(20 \mathrm{ng} / \mathrm{mL})$ plus IFN- $\gamma(10 \mathrm{ng} / \mathrm{mL})$ or IL-4 (20 ng/mL). Simultaneously, the cells stimulated with LPS+IFN- $\gamma$ were co-cultured with specified concentrations of luteolin for $24 \mathrm{~h}$. The cell morphology was observed under an inverted microscope (Olympus, Japan). Cells and culture supernatants were collected for subsequent mRNA and protein analysis.

Quantitative real-time PCR (qPCR). BMDM were stimulated with LPS plus IFN- $\gamma$ or IL-4 respectively, following dosing stimuli, cells were collected and total RNA was extracted by Trizol (Ambion Life Technology, USA) and reverse-transcribed into cDNA. With GAPDH as the internal reference, qPCR was carried out using SYBR Green Master Mix (Toyobo, Japan) according to primer sequences with Quant studio DX real-time quantitative PCR employed biosystems (Life Technologies, USA). Relative gene expression was calculated using the $2^{-\Delta \Delta \mathrm{ct}}$ comparative method. Primer Sequences were obtained from Generay Biotech Co. Ltd. (Shanghai, China) and listed in Table 1.

Table 1. Primers for quantitative real-time PCR

Genes $\quad$ Forward $\left(5^{\prime}-3^{\prime}\right) \quad$ Reverse (5'-3')




\begin{tabular}{|c|c|c|}
\hline CD86 & TCAATGGGACTGCATATCTGCC & GCCAAAATACTACCAGCTCACT \\
\hline iNOS & CAGAGGACCCAGAGACAAGC & TGCTGAAACATTTCCTGTGC \\
\hline IL-6 & TAGTCCTTCCTACCCCAATTTC & TTGGTCCTTAGCCACTCCTTC \\
\hline TNF- $\alpha \square$ & ССТСССТСТСATCAGTTCTA & ACTTGGTGGTTTGCTACGAC \\
\hline Arg1 & CAGAAGAATGGAAGAGTCAG & CAGATATGCAGGGAGTC \\
\hline CD206 & CAGGTGTGGGCTCAGGTAGT & TGTGGTGAGCTGAAAGGTGA \\
\hline IL-10 & CTTACTGACTGGCATGAGGATCA & GCAGCTCTAGGAGCATGTGG \\
\hline GAPDH & TGAAGCAGGCATCTGAGGG & CGAAGGTGGAAGAGTGGGAG \\
\hline
\end{tabular}

ELISA for cytokines secretion. BMDM in exponential phase were inoculated in a six-well plate overnight. Successively, the drugs induced cell polarization and combined with different concentrations of luteolin for $24 \mathrm{~h}$. The levels of cytokines in the supernatants were performed according to commercial ELISA kit (eBioscience, USA) instructions. Logistic fitting-curve for two of four parameters was used to calculate the concentration of cytokines.

Flow Cytometric Staining of BMDM surface Markers. Totally, $5 \times 10^{5}$ BMDM were resuspended in $100 \mu \mathrm{L}$ PBS, then incubated with $0.5 \mu \mathrm{L}$ anti-mouse CD16/32 blocking antibody (BioLegend, San Diego, USA) to avoid nonspecific binding in an ice bath for $20 \mathrm{~min}$. Subsequently, cells were stained with anti-mouse FITC-CD11c $(0.5 \mu \mathrm{L}$; BioLegend) or APC-CD206 (10 $\mu \mathrm{L}$; BioLegend) and protected from exposure to light for $30 \mathrm{~min}$ at room temperature. After washing with PBS, the cells were fixed with $0.5 \mathrm{~mL}$ paraformaldehyde at $4^{\circ} \mathrm{C}$, and the mean fluorescence intensity (MFI) of membrane surface antigen CD11c or CD206 were analyzed by FCM. 
140 Protein extraction and immunoblotting. Collect cells and extract total protein for protein

141 quantification by BCA. After $20 \mu \mathrm{g}$ protein was subjected to SDS-PAGE and transferred to PVDF

142 membrane (millipore, USA), the corresponding primary antibodies against p-STAT1-tyr ${ }^{701}$,

143 p-STAT6-tyr ${ }^{641}$ (Cell Signaling Technology, USA) and $\beta$-actin (Sigma, USA) were applied at $4^{\circ} \mathrm{C}$

144 overnight. Then membranes were washed and incubated with HRP-conjugated secondary

145 antibodies with shaken at room temperature for $30 \mathrm{~min}$. Immunoreactive proteins were exposed

146 and developed using ECL (Beyotime, China), and $\beta$-actin was used as an internal reference to

147 calculate the relative expression of protein.

148 Statistical analysis. The experimental data were presented as mean \pm SD. One-way ANOVA

149 followed by Tukey's post-hoc test was used in the multiple comparisons. Analysis was performed

150 using the GraphPad Prism 5.0 software (San Diego, CA, USA). $P<0.05$ was considered

151 statistically significant.

\section{RESULTS}

Mouse bone marrow cells differentiate into macrophages. The isolated mouse bone 

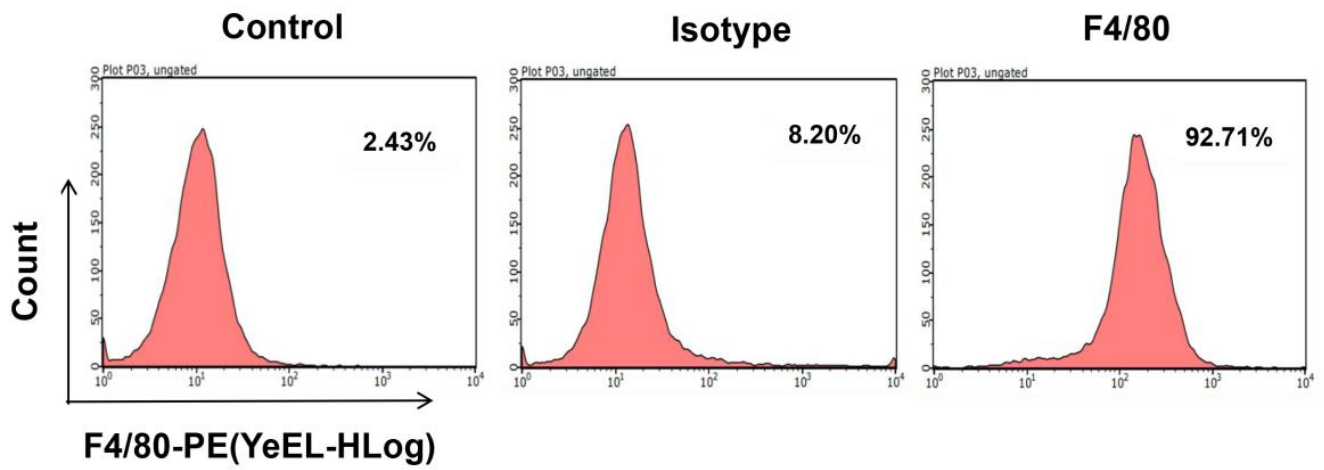

FIG 1 The differentiation proportion of BMDM. FCM detected the surface marker F4/80 of

BMDM after induction for 7 days, and the positive rate was $92.71 \%$.

A<smiles>O=c1cc(-c2ccc(O)c(O)c2)oc2cc(O)cc(O)c12</smiles>

B

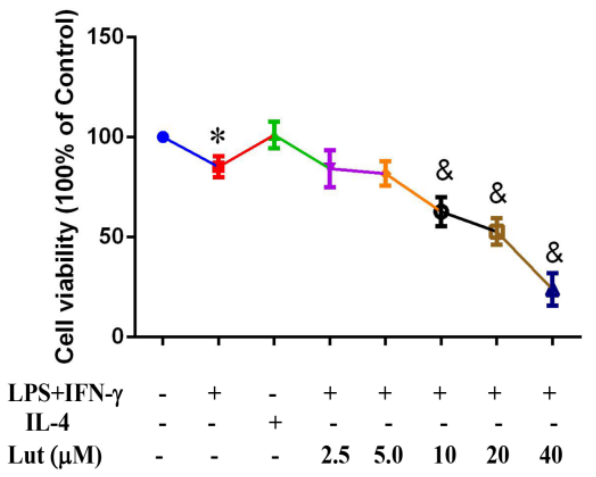

FIG 2 Effect of luteolin on LPS+IFN- $\gamma$-primed BMDM viability. A. Structure of luteolin. B. 
171 according to ANOVA and Tukey test. $* P<0.05$ vs. control group (non-treated control); ${ }^{\&} P<0.05$

172 vs. LPS+IFN- $\gamma$-treated group.

173 Morphology of polarized BMDM. Microscopically, BMDM showed typical morphology of

174 macrophages, such as round, oval or irregular shape, with pseudopods and adherential growth (Fig.

175 3. Control group). After being induced into M1-phenotype by LPS plus IFN- $\gamma$, the BMDM

176 presented oval "Fried egg" appearance and pseudopodia extension (Fig. 3. LPS+IFN- $\gamma$-treated

177 group), while the M2-type BMDM induced by IL-4 were round and plump cytoplasm,

178 accompanied by short pseudopodia (Fig. 3. IL-4-treated group). After various dose of luteolin

179 contribution, M1 cells contracted slightly and pseudopodia became shorter (Fig. 3.

180 LPS+IFN- $\gamma$-combined with 2.5/5.0 Lut-treated groups). 
A

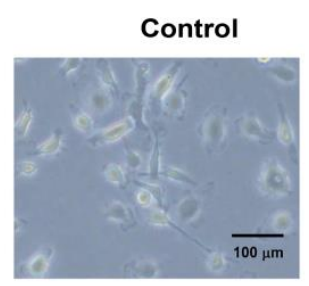

LPS+IFN- $\gamma$
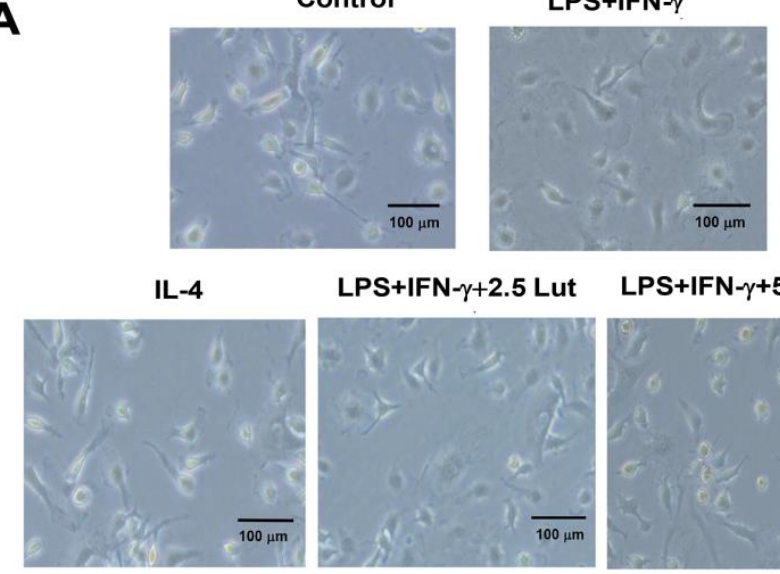

LPS+IFN- $\gamma+5.0$ Lut

B

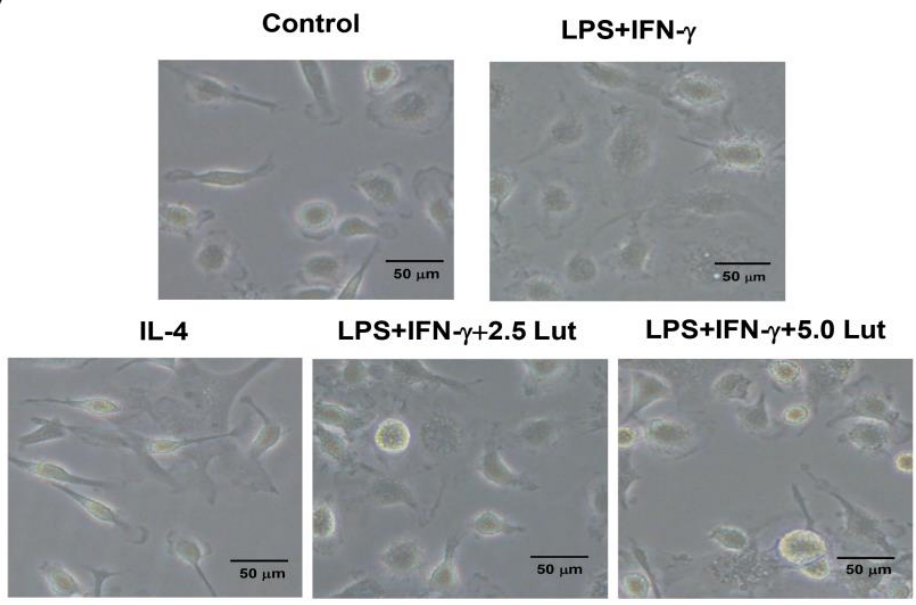

FIG 3 The morphology of polarized BMDM (A. 100x; B. 200x). BMDM were polarized with

LPS+IFN- $\gamma$ or IL-4, simultaneously, BMDM exposed to LPS+IFN- $\gamma$ were administrated with system. 
192

193

194

combined with luteolin, the expression of M1-type pro-inflammatory factors were decreased (Fig.

4), while the expression of M2-type anti-inflammatory factors were increased (Fig. 5).

A

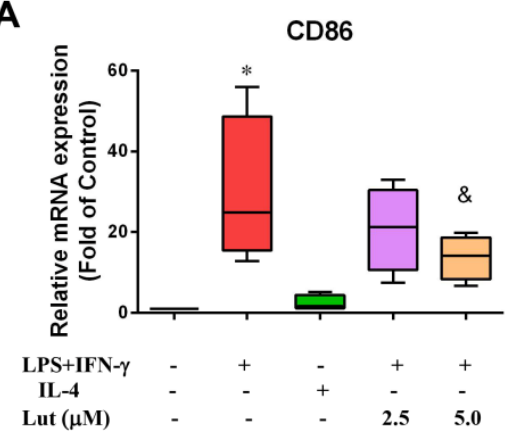

C

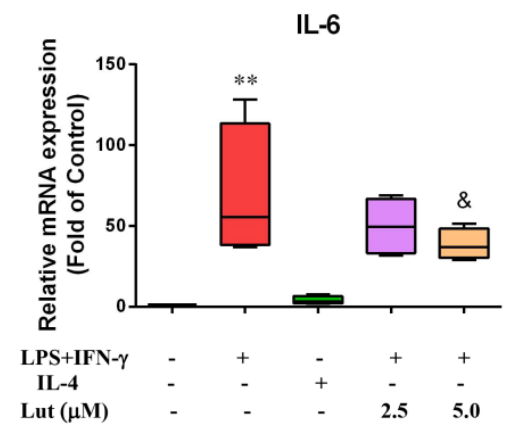

B

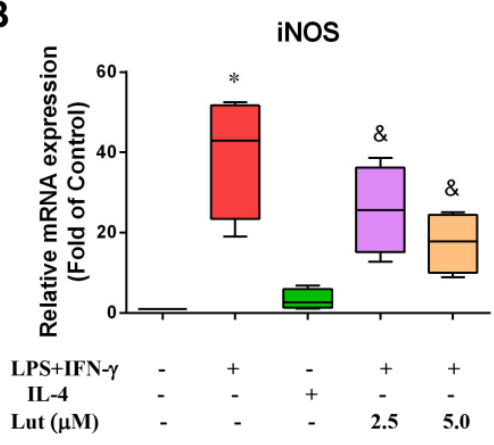

D

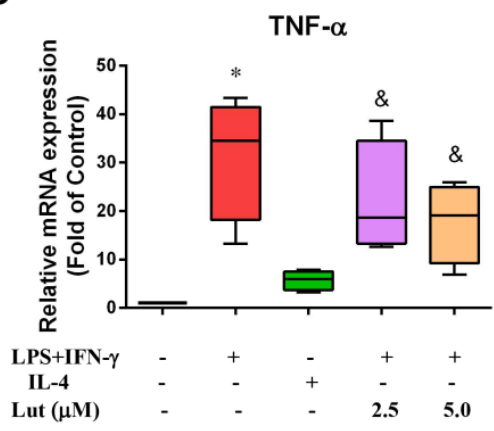

FIG 4 Effect of luteolin on the expression of M1-type pro-inflammatory factors in activated BMDM. The M1-type mRNA molecules were determined by qPCR with GAPDH as an internal control. BMDM were primed with LPS+IFN- $\gamma$ or IL-4, and LPS+IFN- $\gamma$-treated-BMDM $\square$ incubated with indicated doses of luteolin for $24 \mathrm{~h}$, the relative M1-type mRNA levels of CD86 (A), iNOS (B), IL-6 (C) and TNF- $\alpha \square$ (D) in M1-polarized macrophages reduced slowly. Data represented mean \pm SD of four independent experiments performed in duplicate. Different symbols indicate a significant difference according to ANOVA and Tukey test. ${ }^{*} P<0.05,{ }^{*} P<0.01$ vs. control group (non-treated control); ${ }^{\&} P<0.05$ vs. LPS+IFN- $\gamma$-treated group. 
205
A

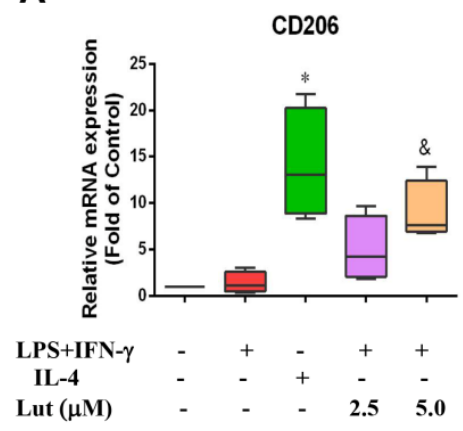

B

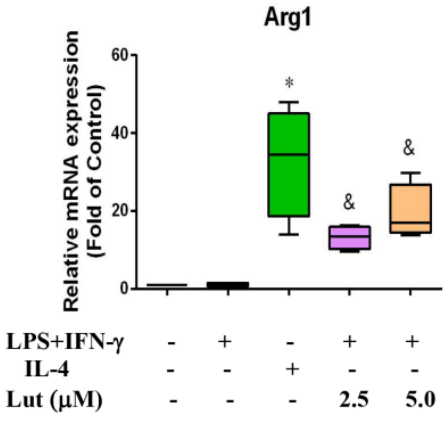

C

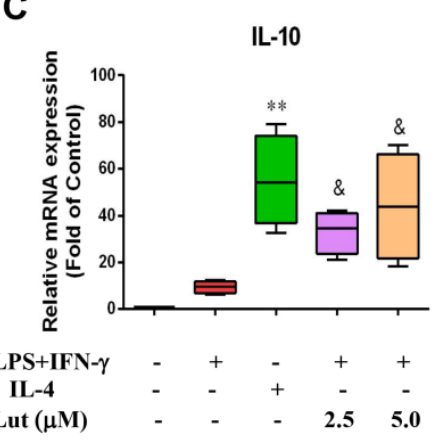

FIG 5 Effect of luteolin on the expression of M2-type anti-inflammatory factors in activated BMDM. The M2-type mRNA molecules were assessed by qPCR with GAPDH as an internal control. BMDM were primed with LPS+IFN- $\gamma$ or IL-4, and LPS+IFN- $\gamma$-treated $\square$ BMDM $\square$ incubated with indicated doses of luteolin for $24 \mathrm{~h}$, the relative M2-type mRNA levels CD206 (A), Arg1 (B) and IL-10 (C) elevated gradually. Data represented mean \pm SD of four independent experiments performed in duplicate. Different symbols indicate a significant difference according to ANOVA and Tukey test. $* P<0.05, * * P<0.01$ vs. control group (without treatment); ${ }^{\&} P<0.05$ vs. LPS+IFN- $\gamma$-treated group.

Effect of luteolin on inflammatory cytokine levels in polarized BMDM. To further explore the polarity skewing effect of luteolin in BMDM, IL-6 and IL-10 production were measured with ELISA. Pro-inflammatory cytokine IL-6 liberated by M1-polarized BMDM increased significantly, in the meantime, anti-inflammatory cytokine IL-10 secreted by M2-polarized BMDM also amplificated clearly, which was statistically different from the control group (without treatment). After various dose of luteolin challenged to M1-polarized BMDM, IL-6 released by M1-polarized BMDM lowered visibly, while IL-10 elevated obviously, compared with corresponding LPS+IFN- $\gamma$-treated group, there was a statistical difference (Fig. 6). 

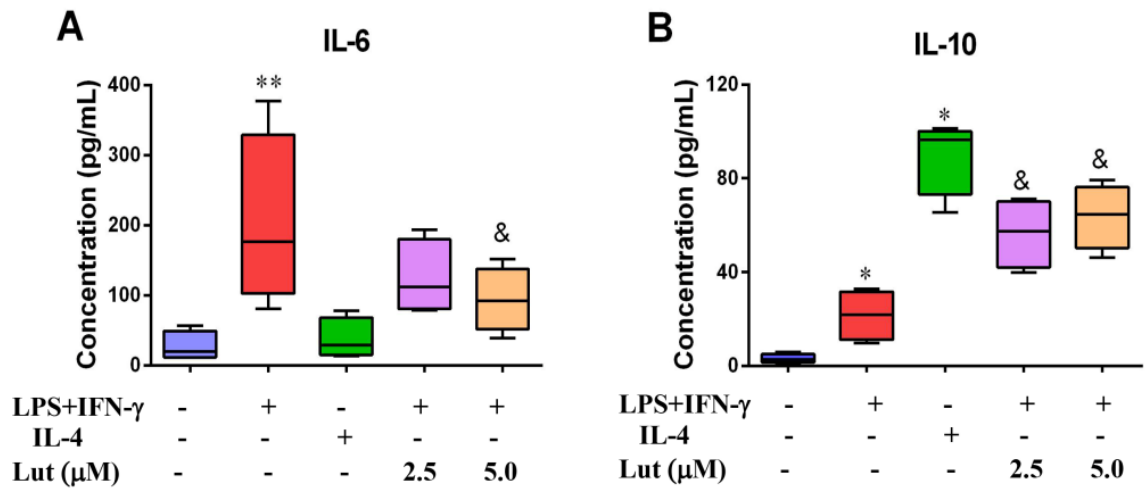

FIG 6 Effect of luteolin on IL-6 and IL-10 levels in polarized BMDM. BMDM were primed with

LPS+IFN- $\gamma$ or IL-4, followed by luteolin exposure for $24 \mathrm{~h}$. Supernatants were harvested and levels of IL-6 (A) and IL-10 (B) secreted from the M-polarized BMDM were measured via ELISA. Data represented mean $\pm \mathrm{SD}$ of four independent experiments performed in duplicate. Different symbols indicate a significant difference according to ANOVA and Tukey test. $* P<0.05$ vs. Control group (without treatment); ${ }^{\&} P<0.05$ vs. LPS+IFN- $\gamma$-treated group. elevated that the MFI of CD11c (60.81) in M1-polarized BMDM was significantly enhanced compared with that of IL-4 treatment group (13.84) and Control group (11.59). The MFI of CD206 (58.36) in M2-polarized BMDM was also significantly amplified than that in LPS+IFN- $\gamma$ alone treatment group (12.46) and Control group (7.33). Against this, luteolin treatment dramatically attenuated the CD11c MFI to 29.56 (LPS+IFN- $\gamma+2.5$ Lut-treated group) and 23.02 (LPS+IFN- $\gamma+5.0$ Lut-treated group) in M1-polarized BMDM, but gradually strengthened the 
A
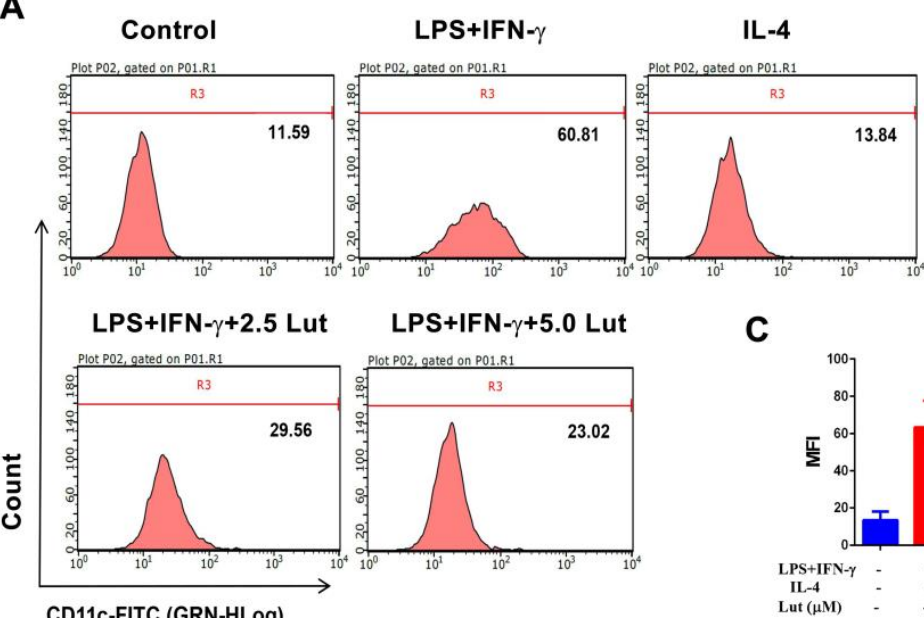

C

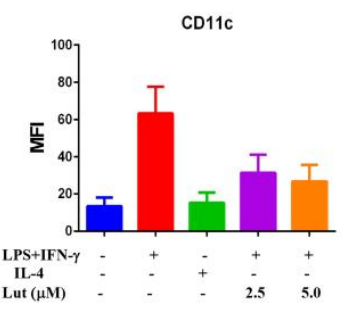

B

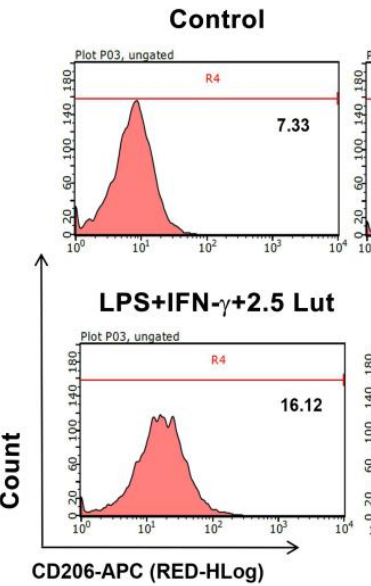

LPS+IFN- $\gamma$

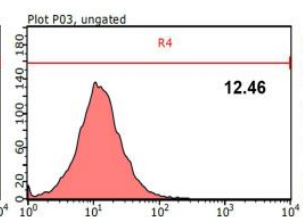

IL-4

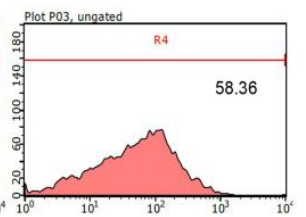

LPS+IFN $-\gamma+5.0$ Lut

D

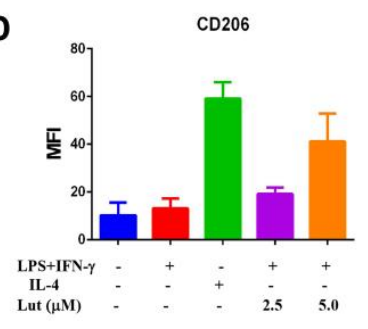

FIE 7 Effects of luteolin on the expression of BMDM surface markers CD11c and CD206. The

BMDM were stimulated with LPS+IFN- $\gamma$ or IL-4, and then luteolin treatment for $24 \mathrm{~h}$, the

expression levels of CD11c (A) and CD206 (B) protein on BMDM are presented as MFI as

Control group (without treatment); ${ }^{\&} P<0.05$ vs. LPS+IFN- $\gamma$-treated group. 
A

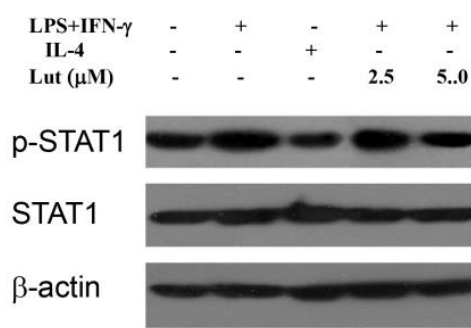

B

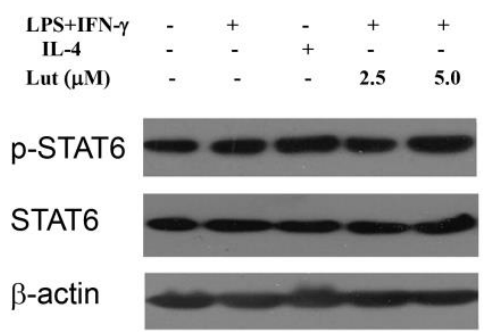

\section{C}

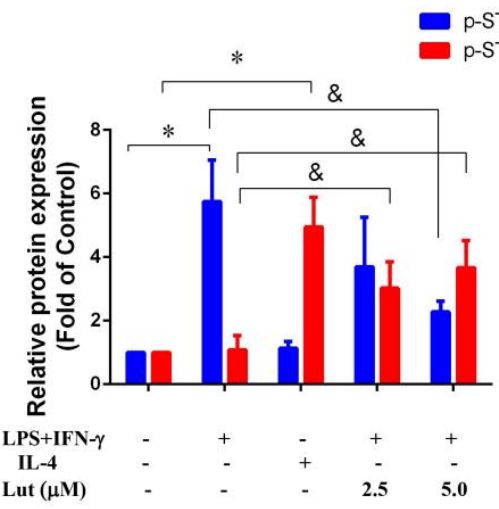

FIG 8 Effects of luteolin on the protein levels of p-STAT1/6 in polarized BMDM. The BMDM were primed with LPS+IFN- $\gamma$ or IL-4, and then for addition of luteolin for $24 \mathrm{~h}$. Total cell lysates were analyzed by immunoblotting for the indicated antibody, respectively. $\beta$-Actin was used as loading control. Representative immunoblots of p-STAT1 (A) and p-STAT6 (B); The relative protein levels of p-STAT1and p-STAT6 (D) by densitometric analysis. Data represented mean \pm SD of three independent experiments. Different symbols indicate a significant difference according to ANOVA and Tukey test. ${ }^{*} P<0.05$ vs. Control group (without treatment); ${ }^{\&} P<0.05$ 


\section{DISCUSSION}

Infectious diseases are the leading cause of death, and the infection severity is due to an

endotoxin, is the outer membrane structure of cell wall of Gram-negative bacteria which can bind

to toll-like receptor 4 (TLR4) on macrophage surface to induce M1 polarization and secrete

cytokines, cause SIRS, and severe cases cause sepsis and MOF (7). Therefore, it is particularly

important to regulate macrophage polarization, avoid excessive activation of M1 macrophages,

reduce inflammation and promote tissue repair. BMDM are suitable cell models for studying

iNOS, TNF, IL-6 and surface markers CD86 and CD11C were up-regulated; IL-4 stimulated

BMDM M2 polarization, and M2-type anti-inflammatory factors including Arg1, IL-10 and 
are one of the pivotal signal transduction pathways and widely involved in the process of cell

Zhou et al. (18) showed that IL-6 and IFN- $\gamma$ released by LPS-polarized M1 macrophages can

its receptor, and simultaneously, the activated STAT1 can further provoke the levels of TNF- $\alpha$,

IL-1 $\beta$ and iNOS in macrophages. iNOS, is a signature of M1-polarized macrophages, which

responsible for nitric oxide (NO) production when cells are stimulated by IFN- $\gamma$ or LPS, and

surface markers of M1 macrophages. CD86 is a B7 costimulatory molecule that stimulates the activation of antigen-presenting cells to secrete more pro-inflammatory factors. In the meantime,

binds to bacterial LPS, which activates $\mathrm{CD}^{+} \mathrm{T}$ cells to proliferate and differentiate into Th1 cells and secrete massive TNF- $\alpha$, IL-6 and IL-12 to trigger inflammatory cascades (22). Two other activated "mononuclear-macrophage system", are elevated not only in bacterial infection but also during viral infection (23-24). More importantly, they are most strong pro-inflammatory agent causing "cytokine storm". Studies have shown that patients with severe COVID-19 characterized 
M2-type anti-inflammatory factors such as IL-10, Arg1 and CD206 are up-modulated. In this regard, IL-4 binds to its receptor to activate JAK to further phosphorylate STAT6 and enhance the Arg1 activity. Arg1 and iNOS are important hallmarkers of M2/M1 type macrophage polarization, respectively. Under normal circumstances, the activities of Arg1 and iNOS are strictly regulated by macrophages and maintain a dynamic equilibrium. When M2 polarization occurs, Arg 1 competes for iNOS to decompose substrate arginine, thus benefit for tissue regeneration. Moreover, Arg1 is also inseparable from M2 macrophage properties in playing immune memory function to eliminate infectious agents. CD206, so called mannose receptor, is a membrane surface marker of M2 cells, which can specifically recognize antigens to clear pathogens, promote angiogenesis and repress immune response (27). Another M2-type anti-inflammatory factor, IL-10, on the one hand, enhances the sensitivity of macrophages to IL-4 and IL-13 by increasing the abundance of IL-4 receptors on the macrophage surface, which contributes to M2-type polarization of macrophages. On the other hand, it can synergize with IL-4 to inhibit pro-inflammatory cytokines IL-1 $\beta$ and TNF- $\alpha$ to reduce inflammation. In the light of preliminary data, IL-10 displayed higher levels in patients with sepsis and serious COVID-19 (28). All these indicate that when inflammation is motivated, an intricate network is formed between the pro-inflammatory mediators and activated STAT1, eliciting "inflammatory storm." Nevertheless, upon luteolin contribution, a complex network is also formed between anti-inflammatory mediators and activated STAT6, further facilitating the expression of anti-inflammatory factors which resist the formation of pro-inflammatory factors and alleviate inflammation accordingly. Herbal compound Physalin D can repolarize M1 toward M2 polarization in BMDM through STAT1 suppression and STAT6 activation (29), which consist with our study. 
pathways. Only by actively exploring the regulation of BMDM polarization and maintaining the

balance of inflammatory mediators can maintain the physical stable. In this investigation,

LPS/IFN- $\gamma$ induced M1 polarization and IL-4 induced M2 polarization of BMDM. After being

pro-inflammatory factors and elevated M2-type anti-inflammatory factor, and that signaling protein p-STAT1 was down-regulated and P-STAT6 was up-regulated. That is, the macrophage

pro-inflammatory to anti-inflammatory. In light of these findings, our research provide a novel insight into the role of luteolin to be a candidate for controlling macrophage phenotype to treat infectious disease.

\section{ACKNOWLEDGEMENTS}

We acknowledge the support of their staff and Facility of department of Endocrine and

(Chen Guofang) edited this manuscript and analyzed data. 


\section{REFERENCES}

1. Chousterman BG, Swirski FK, Weber GF. 2017. Cytokine storm and sepsis disease

2. Greten FR, Grivennikov SI. 2019. Inflammation and cancer: triggers, mechanisms, and

Melatonin as a potential adjuvant treatment. Life Sciences 250 (2020): 117583. management of severe COVID-19. Beyond the anti-viral therapy: a comprehensive review. 

hypocholesterolemic mechanisms in mice fed a high-fat diet. Biomed Pharmacother 96:1000-1007. https://doi: 10.1016/j.biopha.2017.11.131.

10. Yao Y, Rao C, Zheng G, Wang S. 2019. Luteolin suppresses colorectal cancer cell metastasis 
390 chinese medicines in treating coronavirus disease 2019 (COVID-19). Pharmacol Res 158:104939. role in severe dengue. Semi Immunopathol 39: 563-574. https://doi: 10.1007/s00281-017-0625-1.

2008. Amplification of the signal transducer and activator of transcription I signaling pathway and polarization and function with emphasis on the evolving roles of coordinated regulation of cellular signaling pathways. Cell Signal 26: 192-197. https://doi: 10.1016/j.cellsig.2013.11.004. marrow-derived mesenchymal stem cells highly strengthens their potential to promote 
411

412

413

21. Soltys J, Bonfield T, Chmiel J, Berger M. 2002. Functional IL-10 deficiency in the lung of cystic fibrosis (cftr(-/-)) and IL-10 knockout mice causes increased expression and function of B7 costimulatory molecules on alveolar macrophages. J Immunol 168: 1903-1910. https://doi: 10.4049/jimmunol.168.4.1903.

22. Sándor N, Lukácsi S, Ungai-Salánki R, Orgován N, Szabó B, Horváth R, Erdei A, Bajtay Z. 2016. CD11c/CD18 dominates adhesion of human monocytes, macrophages and dendritic cells over CD11b/CD18. Plos One 11:e0163120. https://doi: 10.1371/journal.pone.0163120.

23. Indalao IL, Sawabuchi T, Takahashi E, Kido H. 2017. IL-1 $\beta$ Is a key cytokine that induces trypsin upregulation in the influenza virus-cytokine-trypsin cycle, Arch Virol 162:201-211. https://doi: 10.1007/s00705-016-3093-3.

24. Gubernatorova EO, Gorshkova EA, Polinova AI, Drutskaya MS. 2020. IL-6: relevance for immunopathology of SARS-CoV-2. Cytokine Growth F $\mathrm{R}$ 53:13-24. https://doi: 10.1016/j.cytogfr.2020.05.009.

25. Tufan A, Güler AA, Matucci-Cerinic M. 2020. COVID-19, immune system response, hyperinflammation and repurposing antirheumatic drugs. Turk J Med Sci 50:620-632. https://doi: 10.3906/sag-2004-168.

26. Feldmann M, Maini RN, Woody JN, Holgate ST, Winter G, Rowland M, Richards D, Hussell T. 2020. Trials of anti-tumour necrosis factor therapy for COVID-19 are urgently needed. Lancet 395:1407-1409. https://doi: 10.1016/S0140-6736(20)30858-8.

27. Dai K, Huang L, Sun XM, Yang LH, Gong ZJ. 2015. Hepatic CD206-positive macrophages 
431 express amphiregulin to promote the immunosuppressive activity of regulatory $\mathrm{T}$ cells in HBV

432 infection. J Leukoc Biol 98: 1071-1080. https://doi: 10.1189/jlb.4A0415-152R. 